\title{
An Update on Gnathic Pathology
}

\author{
Angela C. Chi $\cdot$ John M. Wright
}

Received: 17 October 2014/ Accepted: 29 October 2014/Published online: 20 November 2014

(C) Springer Science+Business Media New York 2014

Over the past decade, there have been many exciting developments in the field of gnathic pathology. Thus, for this special issue of Head and Neck Pathology, we have assembled a diverse panel of international experts to share their insights regarding recent advances and emerging issues in pathologic conditions of the jaws.

Of course, no update on gnathic pathology would be complete without a discussion of odontogenic lesions. These rare, distinctive lesions with their vast heterogeneity continue to marvel and challenge us as pathologists. In their editorial, Drs. Wright, Odell, Speight, and Takata raise several thoughtprovoking issues regarding the current World Health Organization (WHO) classification of odontogenic tumors [1]. For example, should this classification scheme include not only odontogenic neoplasms but also odontogenic cysts and allied lesions? Even more fundamentally, how shall we define a tumor, neoplasm, cyst, and cystic neoplasm? Can we form a consensus regarding criteria for malignancy in odontogenic tumors? Furthermore, clarification is needed regarding odontogenic tumors with borderline malignant behavior, and confusion persists regarding a myriad of entities, including unicystic ameloblastoma, odontoameloblastoma, the "simple type" of odontogenic fibroma, and various "types" of ossifying fibroma-just to name a few.

Drs. Richardson and Müller focus specifically on odontogenic malignancies. Among the interesting issues that they discuss is the difficulty of distinguishing ameloblastic

\footnotetext{
A. C. Chi $(\bowtie)$

College of Dental Medicine, Medical University of South

Carolina, Charleston, SC, USA

e-mail: chi@musc.edu

J. M. Wright

Department of Diagnostic Sciences, Texas A\&M University

Baylor College of Dentistry, Dallas, TX, USA
}

carcinoma from so-called "atypical ameloblastoma," with recent investigations suggesting immunohistochemistry for SOX2 and Ki-67 as a useful aid in predicting malignancy [2]. In addition, the recent discovery of the EWSR $1-A T F 1$ translocation in not only hyalinizing clear cell carcinoma of salivary glands but also clear cell odontogenic carcinoma poses interesting questions regarding appropriate classification [3]. Also, there is a need for further characterization of sclerosing odontogenic carcinoma, which was first reported after the 2005 WHO classification [4]. Moreover, Dr. MosquedaTaylor and colleagues present evidence for another new entity, termed "odontogenic carcinoma with dentinoid," which also deserves consideration for inclusion in the next WHO classification.

With the advent of a new millennium, we have experienced a remarkable evolution in molecular pathology, which has paved the way for a new understanding of human disease and has had significant implications for classification, diagnosis, treatment, and prognosis. However, our understanding of the molecular genetics of odontogenic lesions is still relatively nascent. Dr. Bilodeau and colleagues review recent molecular genetic findings in odontogenic lesions and provide guidance on practical clinical applications, where applicable. The identification of BRAF V600E and SMO mutations in ameloblastoma and the EWSRI-ATF1 in clear cell odontogenic carcinoma are among the most exciting recent discoveries, although clearly much more research is needed in this arena. In addition, Drs. Hunter and Speight provide their insights regarding the practical utility of immunohistochemistry for the diagnosis of odontogenic lesions.

Despite advances in molecular diagnostics and immunohistochemistry, meticulous correlation of histomorphology with clinical, imaging, and laboratory findings still forms the backbone of gnathic and, more generally, bone pathology. Accordingly, Dr. Benson and colleagues review 
recent technologic advances in diagnostic imaging of the jaws and associated tissues. Some of the developments discussed include the rise of digital radiography; the increased availability of cone-beam computed tomography (CBCT); a new generation of computed tomography (CT); the expansion of magnetic resonance (MR) protocols; the latest innovations in nuclear imaging; and current investigations in oral ultrasonography. The advantages, disadvantages, and potential applications for assessment of pathologic conditions of the oral and maxillofacial region are aptly discussed.

Certainly, the range of pathologic conditions of the jaws extends beyond the realm of odontogenic lesions. Dr. ElMofty presents a concise but comprehensive and up-to-date review of benign fibro-osseous lesions of the craniofacial skeleton, and Drs. Flanagan and Speight review giant cell lesions of the jaws, including recent molecular findings. Also, Dr. Stewart and colleagues provide a timely update on select bone- and cartilage-forming tumors and Ewing sarcoma, with an emphasis on gnathic involvement. As investigators of the largest studies to date regarding metastases to the oral cavity, Dr. Hirshberg and colleagues provide an expert review of this topic, including a discussion of malignancies of unknown origin. Furthermore, Dr. Raubenheimer and colleagues examine recent advances in our understanding of metabolic bone diseases that may affect the jaws and share their specialized expertise in quantitative bone histomorphometry. Finally, Dr. Hellstein rounds out this special issue with a contemporary update on osteochemonecrosis of the jaws, which has emerged as an important issue for pathologists and clinicians alike.
In closing, we would like to thank all of our invited experts, their esteemed colleagues, and tireless support staff for their contributions. Without them, this special edition would not have been possible. Also, we would like to express our gratitude to the editors-in-chief, Drs. Susan Müller and Lester D.R. Thompson, for providing their guidance and bestowing upon us the honor and privilege of working on this momentous project. Because of practical limitations, we were not able to provide an update of every pathologic condition involving jaws. However, we have aimed to highlight the most remarkable and fascinating findings to emerge in recent years, and we look forward to the exciting developments that surely will follow in ensuing decades.

\section{References}

1. Barnes L, Eveson JW, Reichert P, Sidransky D, editors. World Health Organization Classification of Tumours. Pathology and genetics. Head and neck tumours. Lyon: IARC Press; 2005.

2. Lei Y, Jaradat JM, Owosho A, et al. Evaluation of SOX2 as a potential marker for ameloblastic carcinoma. Oral Surg Oral Med Oral Pathol Oral Radiol. 2014;117:608-16.

3. Bilodeau EA, Weinreb I, Antonescu CR, et al. Clear cell odontogenic carcinomas show EWSR1 rearrangements: a novel finding and a biological link to salivary clear cell carcinomas. Am J Surg Pathol. 2013;37:1001-5.

4. Koutlas IG, Allen CM, Warnock GR, Manivel JC. Sclerosing odontogenic carcinoma: a previously unreported variant of a locally aggressive odontogenic neoplasm without apparent metastatic potential. Am J Surg Pathol. 2008;32:1613-9. 